\title{
Calculation Model and Decoupling Coefficient Sensitivity Study of Periphery Hole for Eccentric Decoupled Charge in Highway Tunnels
}

\author{
Yuanqing Wang ${ }^{1}$ and Junfeng Liu $\mathbb{D}^{2}$ \\ ${ }^{1}$ The 5th Engineering Ltd., The 11th Engineering Bureau of China Railway, Chongqing 400037, China \\ ${ }^{2}$ School of Civil Engineering, Chongqing University, Chongqing 400045, China \\ Correspondence should be addressed to Junfeng Liu; jfliu1990@foxmail.com
}

Received 27 December 2017; Revised 23 March 2018; Accepted 5 April 2018; Published 17 May 2018

Academic Editor: Cristina Castejón

Copyright (c) 2018 Yuanqing Wang and Junfeng Liu. This is an open access article distributed under the Creative Commons Attribution License, which permits unrestricted use, distribution, and reproduction in any medium, provided the original work is properly cited.

\begin{abstract}
On the basis of the eccentric decoupled charge form on site and borehole concentric decoupled computational theory, the calculation model of eccentric decoupling charge would be established in the present study. The calculation model takes into account the influence factors such as damage to the surrounding rock, air, and water decoupled media. Besides, based on the proposed calculation model, blasting effect of a small-diameter EJ-102 emulsion explosive is calculated by using ANSYS LS-DYNA. The influence of decoupling coefficient, different rock grade, and different RQD value on blasting effect is obtained by sensitivity analysis of the simulated parameters. Then the calculated results are compared with the empirical results. The blasting parameters of fractured rock mass are also optimized by numerical simulation.
\end{abstract}

\section{Introduction}

Overexcavation is a common phenomenon in the blasting construction of highway tunnel so far. The overexcavation has a serious negative effects on the structural stability of the rock mass around the cavern $[1,2]$. Besides, the overexcavation of road tunnel will inevitably affect the field construction of tunnel project $[3,4]$. When the smooth blasting layer is thin, the blast split will occur before the smooth perforative cracks between the peripheral holes, and the clef surface formed inside the rock mass at the same time, which will lead to overexcavation [5-7].

In order to avoid or alleviate the overexcavation phenomenon, the decoupled charge is always adopted for most tunnel periphery hole charge methods $[8,9]$. Since the diameter of stick dynamite is generally smaller than that of the blast-hole, stick dynamite will be eccentric to the hole as the gravity force. The eccentric decoupled charge structure is formed then [1-3]. Wei [10] analyzed the eccentric decouple charge structure theoretically and considered that the crack is firstly produced at the contact point between the explosive and hole wall, and then it played an inhibitory role in the initiation of other points. Liu and Li [11] deduced the calculation formula of eccentric decouple charge, and the result was verified by comparison with the actual engineering application. Besides, Yu et al. [12] studied the explosion characteristics of water medium eccentric decouple charge and finally obtained the optimum formula of charge position. $\mathrm{Pu}$ [13] studied the cement mortar model test of eccentric decouple charge and concentric charge and shows that the initial eccentric stress field is formed on the surrounding rock of the borehole by an eccentric decoupling charge. Furthermore, Zhang [14] studied the explosive stress field distribution of the eccentric decoupled charge structure by means of holographic dynamic photoelastic test. The results show that the charge structure has a great influence on the stress field of the borehole. Zhang et al. [15] carried out field tests on the smooth blasting of eccentric decoupling charge. The results show that the initial pressure of the charging hole is $5 \sim 10$ times away from the charging side. Guan et 


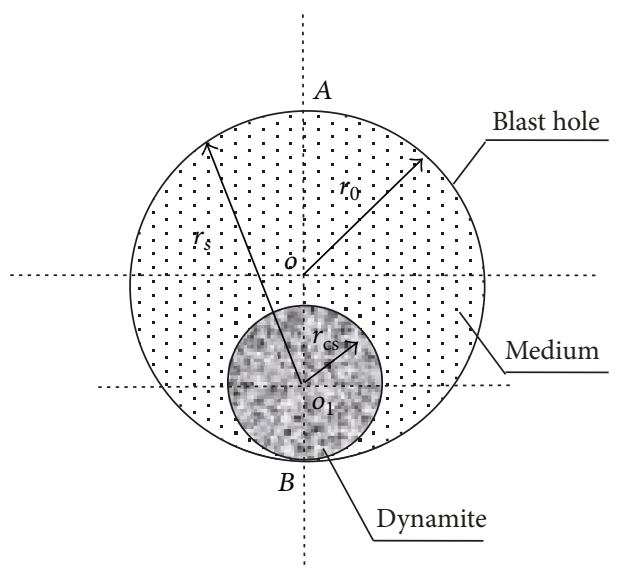

Figure 1: Calculation diagram of eccentric decouple charge, where $o$ is the geometrical center of the hole, $o_{1}$ is the geometrical center of the stick dynamite, $r_{0}$ is the radius of blast-hole, $r_{s}$ is the straight-line distance from the center of the stick dynamite to the wall of blast-hole, and $r_{c s}$ is the radius of stick dynamite.

al. [16] used plexiglass to investigate the crack propagation law of eccentric decoupled charge and they found that when the eccentricity decoupling coefficient is 1.71 , the difference of crack reaches the maximum between the coupled and decoupled sides. Shi et al. [17] carried out field and laboratory tests on mortar specimens; the results suggest the initial pressure of the hole at the charging side of the blast-hole is much larger than that of the noncharging side. Ханукаев [18] pointed out that the heterogeneity, isotropy, own defects, joints, and faults of the rock mass have great influence on the performance of rock blasting. Fourney et al. [19] also carried out further research on eccentric decoupling charge. However, these studies assume that the surrounding rock of the borehole is homogeneous and isotropic, without considering the heterogeneity of the surrounding rock, the initial damage of the surrounding rock, and the damage of surrounding rock caused by the blasting. At the same time, the influence of the decoupling medium of the borehole is not considered.

In the present study, the calculation model of eccentric decoupling charge is established based on the eccentric decoupled charge form on site and borehole concentric decoupled computational theory. It should be noted that the present calculation model takes into account the influence of the factors including damage to the surrounding rock, air, and water decoupled media. Using ANSYS LS-DYNA, the proposed calculation model is applied to several case studies. Finally, the calculated results are compared with the empirical results and the blasting parameters of fractured rock mass are optimized by numerical simulation.

\section{Calculation Model of Peripheral Hole Eccentric Decoupled Charge}

Figure 1 shows the main influence factors of the eccentric decouple charge, which are the radius of blast-hole $r_{0}$, the stick dynamite radius $r_{c s}$, and the type of noncoupling medium.

The stress field of surrounding rock with eccentric decouple charge is studied based on the theory of concentric noncoupling charge. The stress field is symmetrized to the stick dynamite center and the adjacent holes. By analysing the stress filed of wave stress, it can be obtained that stress wave field distributes and spreads symmetrized to blast-hole and stick dynamite. As presented in Figure 1, the hole pressure is changed with the variation of the roll center hole wall and radial distance, and the pressure of the wall $\left(P_{\mathrm{H}}\right)$ for the blasthole is calculated based on the concentric noncoupling charge blasting by

$$
P_{\mathrm{H}}=P_{0} \times\left(\frac{r_{0}}{r_{c s}}\right)^{-k},
$$

where $P_{\mathrm{H}}$ is the peak value of the initial incident pressure of surrounding rock, $P_{0}=\rho_{e} D^{2} / 8$ is initial average detonation pressure, $r_{0}$ and $r_{c s}$ are the radius of the blast-hole and the dynamite stick, respectively, $D$ and $\rho_{e}$ stand for the bursting speed and density of the explosive material, $k$ is the parameter related to the decoupled medium, and the value of $k$ is taken as 6 and 0.72 for air and water, respectively $[20,21]$.

The pressure value in the hole with the eccentric coupling can be obtained according to the calculation formula of hole wall pressure at the blasting of concentric decoupled charge in this paper. The following can be obtained based on (1):

$$
\begin{aligned}
& P_{c s}=P_{0} \cdot\left[\frac{r_{c s}}{r_{s}}\right]^{k}=\left\{\begin{array}{l}
P_{0} \cdot\left[\frac{r_{c s}}{r_{s}}\right]^{0.72} \quad(\text { water }) \\
P_{0} \cdot\left[\frac{r_{c s}}{r_{s}}\right]^{6} \quad(\text { air }),
\end{array}\right. \\
& r_{s}=\sqrt{r_{0}^{2}+\left(r_{0}-r_{c s}\right)^{2}-2 \cdot r_{0} \cdot\left(r_{0}-r_{c s}\right) \cdot \cos (\pi-\theta)}
\end{aligned}
$$




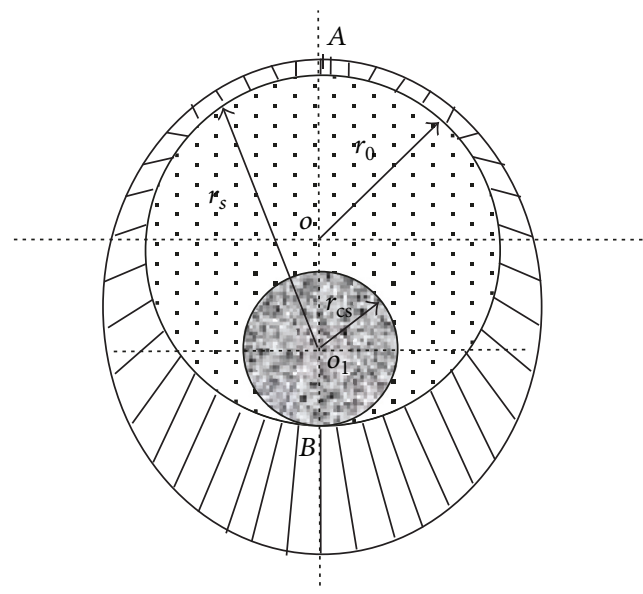

FIGURE 2: Initial blast-hole pressure value diagram of eccentric decouple charge structure.

Combining (2) and (3), it can be derived that

$$
P_{c s}=P_{0} \cdot\left[\frac{r_{c s}}{r_{s}}\right]^{k}=\left\{\begin{array}{l}
\frac{\rho_{e} D^{2}}{2 \cdot(\kappa+1)} \cdot\left[\frac{r_{c s}}{\sqrt{r_{0}^{2}+\left(r_{0}-r_{c s}\right)^{2}-2 \cdot r_{0} \cdot\left(r_{0}-r_{c s}\right) \cdot \cos (\pi-\theta)}}\right]^{0.72} \quad \text { (water) } \\
\frac{\rho_{e} D^{2}}{2 \cdot(\kappa+1)} \cdot\left[\frac{r_{c s}}{\sqrt{r_{0}^{2}+\left(r_{0}-r_{c s}\right)^{2}-2 \cdot r_{0} \cdot\left(r_{0}-r_{c s}\right) \cdot \cos (\pi-\theta)}}\right]^{6} \quad \text { (air), }
\end{array}\right.
$$

where $P_{c s}$ is the initial pressure of the surrounding rock with eccentric decoupling charge. Then based on (4), the distribution of the bore pressure can be obtained (see Figure 2).

It can be found that inside the blast-hole, the pressure value is larger in the side of the stick dynamite. As presented in Figure 2, the pressure in $B$ point reaches the maximum value, and the pressure of $A$ point away from the loading side is smaller, which reaches the minimum value.

The distribution of the incident pressure of the surrounding rock can be calculated by the following methods $[12,14]$ :

$$
P_{b}=n \cdot P_{c s},
$$

where $P_{b}$ is the initial transmission pressure value of surrounding rock and $n$ is the power augmentation factor, which is generally taken between the values of 8 and 11 .

The variation law of radial stress amplitude and circumferential stress amplitude of blast stress wave is as follows [22]:

$$
\begin{aligned}
& \left(\sigma_{r}\right)_{\max }=p_{d}\left(\frac{0.5 d}{r}\right)^{\alpha}+\sigma_{r 0}(r), \\
& \left(\sigma_{\theta}\right)_{\max }=-p_{d} b\left(\frac{0.5 d}{r}\right)^{\alpha}+\sigma_{\theta 0}(r), \\
& b=\widehat{b}= \begin{cases}-1 & \text { (Shock wave action zone) } \\
\frac{0.8 \mu_{s}}{\left(1-0.8 \mu_{s}\right)} & \text { (Non shock wave action zone), }\end{cases}
\end{aligned}
$$

$$
\begin{aligned}
& \alpha \begin{cases}3 & \text { (Shock wave action zone) } \\
\left(1-D_{0}\right)^{0.5} \rho_{0} \widehat{c} \lambda_{1} \lambda_{3}+\lambda_{2} \lambda_{3} & \text { (Non shock wave action), }\end{cases} \\
& D_{0}=1-\left(\frac{E_{d 0}}{\widehat{E}_{d}}\right)
\end{aligned}
$$

where $b$ is the lateral pressure coefficient of stress wave under the damage condition, $d$ is the diameter of the blasthole, $\sigma_{r 0}(r)$ is the initial stress of surrounding rock, $\widehat{c}$ is the longitudinal wave velocity under lossless condition, $E_{d 0}$ and $\widehat{E}_{d}$ are the initial elastic modulus and the undamaged elastic modulus of the rock mass, respectively, and $\lambda_{1}=-4.11 \times 10^{-8}$, $\lambda_{2}=2.92, \lambda_{3}=0.5 \sim 0.8$ are the constants.

Meanwhile, when the decoupled medium is water, due to the formation of explosion water vapor, the stress field of surrounding rock should also be considered:

$$
\begin{aligned}
p_{w}(r) & =p_{c s} \cdot\left(\frac{r_{s}}{r}\right)^{2}=p_{c s} \\
& \left(\frac{\sqrt{r_{0}^{2}+\left(r_{0}-r_{c s}\right)^{2}-2 \cdot r_{0} \cdot\left(r_{0}-r_{c s}\right) \cdot \cos (\pi-\theta)}}{r}\right)^{2} .
\end{aligned}
$$

The criterion for the radius of rock failure zone is determined by formula (1) (3). Moreover, bringing (6) and 


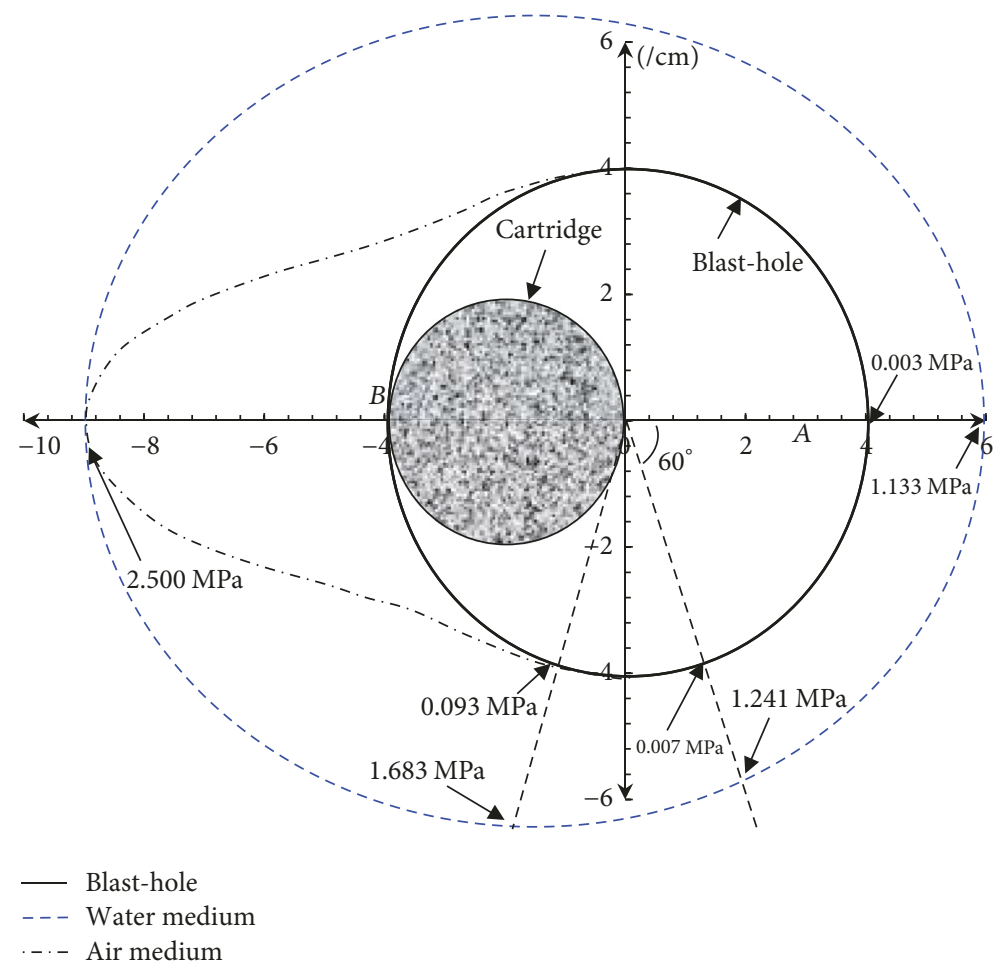

FIGURE 3: Initial blast-hole pressure value diagram of eccentric decouple charge structure when the charge radius is $20 \mathrm{~mm}$, in which the dashed line and the dotted line are the inner pressure distribution of the blast-hole for the decoupling medium of water and air, respectively.

(7) into the criterion, the distribution of crushing zone of surrounding rock with an eccentric decoupled charge structure can be obtained.

\section{Example Analysis}

In this section, the blasting field test in Luo Jiang high-speed road, China, is taken as an example to be analyzed. The level of surrounding rock in the blasting field test is level II, and the lithology is sandstone. The blasting parameters are listed as follows: the hole length $l$ is $4 \mathrm{~m}$, the emulsion explosive and the detonation velocity of explosive are $D=$ $4000 \mathrm{~m} / \mathrm{s}, \rho_{e}=1.25 \times 10^{3} \mathrm{~kg} / \mathrm{m}^{3}$, charge radius $r_{0}$ is $0.02 \mathrm{~m}$, density of rock $\rho_{c}$ is $3.8 \times 10^{3} \mathrm{~kg} / \mathrm{m}^{3}$, compressive strength of surrounding rock $\sigma_{j c}$ is $40 \mathrm{MPa}$, and tensile strength $\sigma_{l}$ is $2.91 \mathrm{MPa}$, Poisson's ratio $v$ is 0.2 , and longitudinal wave velocity of surrounding rock $C_{p}$ is $4390 \mathrm{~m} / \mathrm{s}$. Air density $\rho_{a}$ is $1.29 \mathrm{~kg} / \mathrm{m}^{3}$, water density $\rho_{w}$ is $1.0 \times 10^{3} \mathrm{~kg} / \mathrm{m}^{3}, t_{1}=1 \mathrm{~ms}$ [23], and $t_{3}=8 \mathrm{~ms}$ [23]; supposing that $t_{4}=10 \mathrm{~ms}$ [24], crack length is taken as $0.001 \mathrm{~m}$. The crack angle $\beta$ is taken as $45^{\circ}$.

3.1. Distribution of Surrounding Rock Pressure. The incident pressure of surrounding rock is calculated and analyzed in this part.

As shown in Figure 3, the charging hole radius is $40 \mathrm{~mm}$, and the cartridge radius is $20 \mathrm{~mm}$. In addition, the EJ-102 emulsion explosive is adopted. In the form of an eccentric decoupled charge, the water and air are used in the decoupled media, and the calculated results of the incident pressure of the surrounding rock are shown in Figure 3. It can be seen that, for both water decoupling charge and air decoupling charge, the pressure inside the blast-hole increases from the far side to the near side of the charging site. It should be noted that, for water decoupling charge, the hole pressure distribution is more uniform. It can be seen from Figure 3 that the maximum value of the surrounding rock pressure of $2.5 \mathrm{MPa}$ can be achieved at $B$ point. Comparing the water decoupling medium charge and the air decoupling medium charge, we can also find that the pressure of the surrounding rock under the water decoupling medium charge is obviously larger than that of the air decoupling charge. Specifically, Point $A$, which is in the most distal lateral wall rock, reached a maximum ratio of 377.7 times (1.133/0.003). It can also be seen from Figure 3 that, from the charge side $B$ to the noncharge side $A$, the surrounding rock pressure for air coupling medium owns faster decay ratio than that for water decoupling charge case. Thus, according to Figure 3, the transmission effect of the water decoupling medium charge on the blasting energy is better than that of the air decoupling medium.

Figure 4 shows the distribution of the internal pressure when the water medium is eccentrically loaded. It can be seen from the diagram that the initial internal pressure value increases continuously with the increase of charge radius. Because Point $B$ is a coupling charge, the initial entrance pressure value of the hole reaches the maximum value of $2.5 \mathrm{MPa}$. On the contrary, the incident pressure of the surrounding rock is the minimum. With the decrease of charge diameter, the difference of incident pressure between 


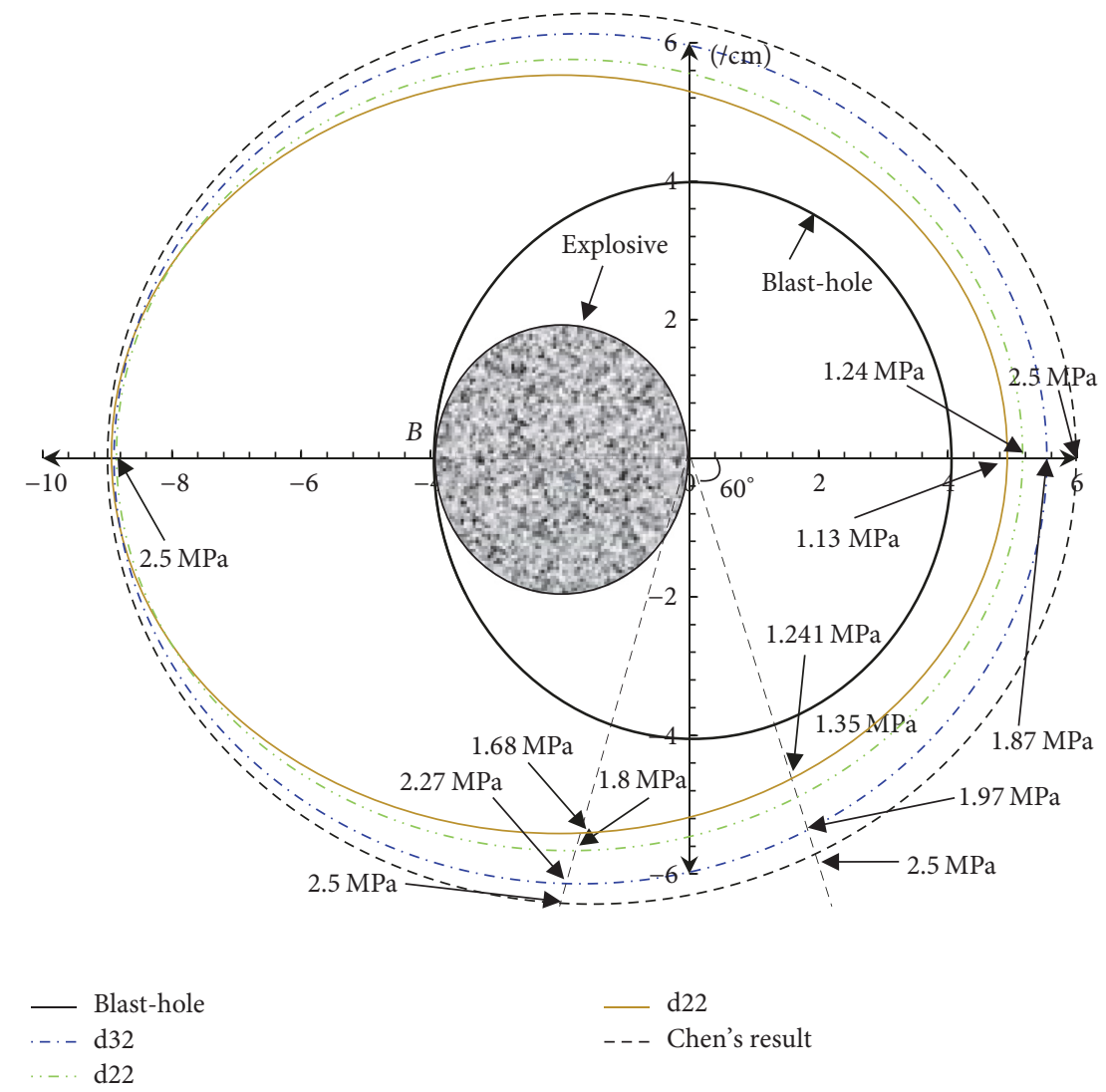

FIGURE 4: The blast-hole pressure value along with the change of charge radius when the decoupling medium is water, where " $d$ " represents the diameter of the explosive and the dashed line stands for the corresponding inner pressure distribution of the blast-hole.

Point $B$ and Point $A$ is increasing. Figure 5 is the variation of the initial pressure value of the hole for different charge diameters in the case of eccentric charge in the air medium. It can be seen that the smaller the charge diameter is, the faster the initial pressure of the surrounding rock will decay from Point $B$ of the loading side to Point $A$ at the nonloading side. Similarly, because Point $B$ is the coupling charge point, the initial pressure value of the surrounding rock of this point is unchanged, and the maximum value is $2.5 \mathrm{MPa}$. In addition, the calculating results of the present model are also in agreement with Chen et al.s research [22].

\subsection{Distribution of Fractured Zone of Surrounding Rock. A} total of four conditions of fractured zone for the surrounding rock are compared and discussed as follows in this section: Conditions 1: the results of this paper for water decoupling charge; Conditions 2: the results of this paper for air decoupling charge; Conditions 3: the calculation results of literature [22] for water decoupling charge; Conditions 4: the calculation results of literature [22] for air decoupling charge.

Figure 6 is a comparison between the theoretical calculation results of the present study and the results of literature [22] when the charge radius is $20 \mathrm{~mm}$ and the eccentric decouple coefficient is 2 . By contrast, it can be found that the radius of the fracture zone formed by water medium charge is larger than that of the air medium charge, which is also in agreement with the result of literature [22]. For air decoupling charge, both the result of literature [22] and the numerical results indicate that the fracture zone formed mainly in the region of charge side and account for about 12 15 times of the blast-hole diameter, whereas, for water decoupling charge condition, distribution of the rupture zone around the blast-hole is more uniform, and the fracture zone accounts for about 15 23 times of the blast-hole diameter. The phenomenon above is for the reason that better transferring effect of the blasting energy is produced for water decoupling charge. Meanwhile, it can also be believed that the blast synergistic effect of water decoupling charge is more obvious than that for air decoupling charge.

\section{Numerical Model}

This section is based on the calculation model for the threephase decoupling calculation model of gas, solid, and water. The $\mathrm{cm}$-g-us unit system is adopted in the simulation model. The model size is $400 \mathrm{~cm} * 460 \mathrm{~cm}$ with $300 \mathrm{~cm}$ hole depth and $4 \mathrm{~cm}$ hole diameter. The specific schematic diagram of the model is depicted in Figure 7. The simulation scheme adopts the form of reverse detonation. In view of the fact that the surrounding rock mass is semi-infinite, the nonreflecting boundary condition is used for the right and left boundaries and the bottom boundary of the numerical model, and the top is a free surface. The actual blasting surrounding rock is an axisymmetric three-dimensional model, which is 



FIGURE 5: The blast-hole pressure value along with the change of charge radius when the decoupling medium is air, where " $d$ " represents the diameter of the explosive and the dashed line stands for the corresponding inner pressure distribution of the blast-hole.

simplified by two-dimensional calculation model to facilitate calculations. Referring to the previous scholars' research, the duration of shock wave, explosion gas, and explosion gas pressure after explosion is set to 300 us. Using ANSYS LSDYNA and LS-PrePost-4.0, the model is preprocessed and after processed, respectively.

The simulation of the fractured zones in the rock mass is introduced as follows. The preprocessing function of ANSYS is employed to simulate the fracture zones such as the joints and the flaws in the rock mass. To begin with, the position of the work plane is changed according to the actual joint orientation and direction, and the joint faces are cut in the analyzed geometric object, which is the necessary condition to produce the joint face. Then take advantage of the multiple loop command of ANSYS to assign different types of physical mechanics parameters to the model. Then the diversity of complexed structures for the established geometric object is reflected. Finally, the types of the units are chosen in the structural model framework formed in ANSYS. Meanwhile, the units of the geometric object are divided according to the joint development characteristics. Then the fractured zones inside the rock mass are formed, which is shown in Figure 7.

\subsection{The Selection of Blasting Materials}

4.1.1. Calculation Model Selection of Explosive Materials. The simulated explosive is made of HIGH_EXPLOSIVE_BURE material model and JWL equation of state, and the equation is [25]

$$
\begin{aligned}
P= & A\left(1-\frac{\omega}{R_{1} V}\right) e^{-R_{1} V}+B\left(1-\frac{\omega}{R_{2} V}\right) e^{-R_{2} V} \\
& +\frac{\omega E_{0}}{V},
\end{aligned}
$$

where $P$ is the hole pressure value, $A=214.4 \mathrm{GPa}, B=$ $0.182 \mathrm{GPa}, R_{1}=130, R_{2}=0.90$, and $\omega$ is the Green Elson parameters, which is taken as $0.15 . V$ is the relative volume of detonation products, and $E_{0}$ is the initial specific energy of the explosive, which is $3.15 \mathrm{GPa}$.

4.1.2. The Material Model of Water and Air Bag. Grunneisen state equation is used for water and air [25]:

$P$

$$
\begin{aligned}
= & \frac{\rho_{0} C^{2} \mu\left[1+\left(1-\gamma_{0} / 2\right) \mu-(a / 2) \mu^{2}\right]}{\left[1-\left(S_{1}-1\right) \mu-S_{2}\left(\mu^{2} /(\mu+1)\right)-S_{3}\left(\mu^{3} /(\mu+1)^{2}\right)\right]} \\
& +\left(\gamma_{0}+a \mu\right) E_{0},
\end{aligned}
$$

where $P$ is pore pressure and $\rho_{0}$ is the density of the material, which is $1 \mathrm{~g} / \mathrm{cm}^{3}$ for water and $0.001252 \mathrm{~g} / \mathrm{cm}^{3}$ for air. $\gamma_{0}$ is coefficient of Grunneisen, which is 0.35 for water and 1.4 for 


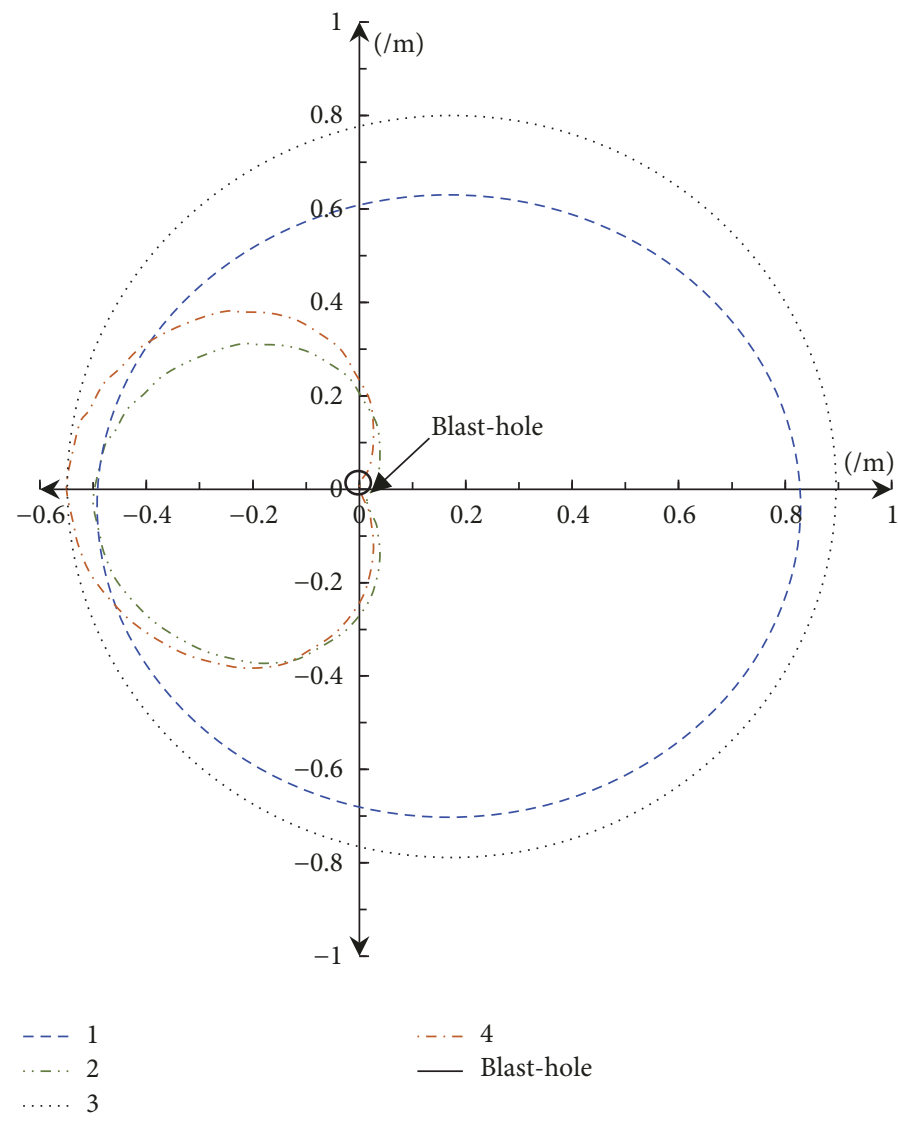

FIGURE 6: The blast-hole rupture zone when the charge radius is $20 \mathrm{~mm}$, where " 1 " and " 3 " stand for the rupture zone around the blast-hole of the present study and Chen et al.s result when the decoupling medium is water, and " 2 " and " 4 " stand for the rupture zone around the blast-hole of the present study and Chen et al's result when the decoupling medium is air.

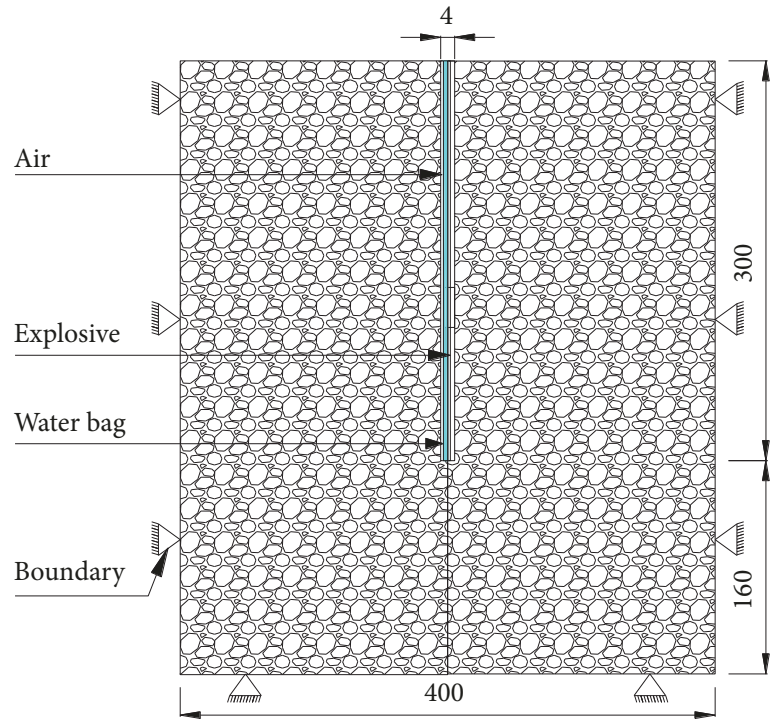

(a) Eccentric decoupling charge

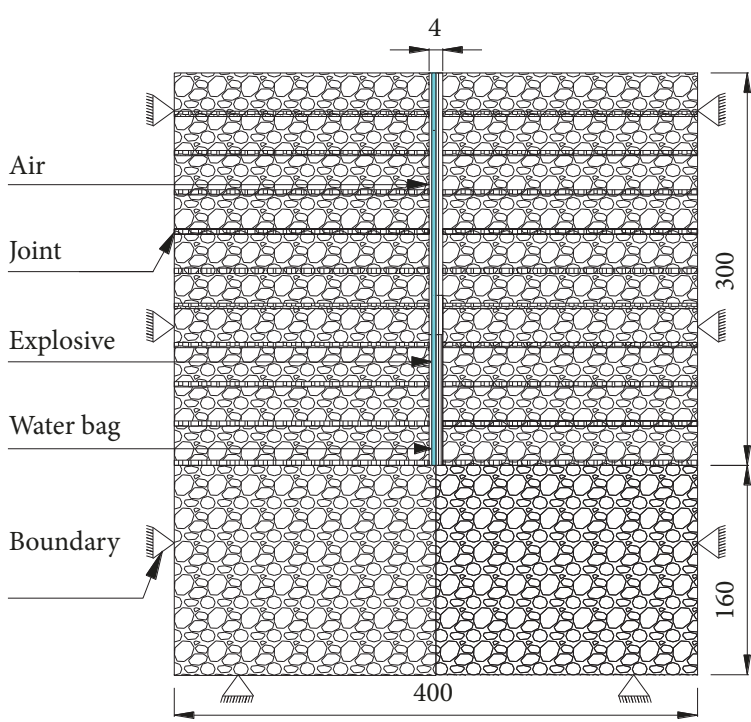

(b) Eccentric uncoupling charge under horizontal joints

FIGURE 7: The numerical simulation calculation model sketch of eccentric decouple charge (unit/cm). 


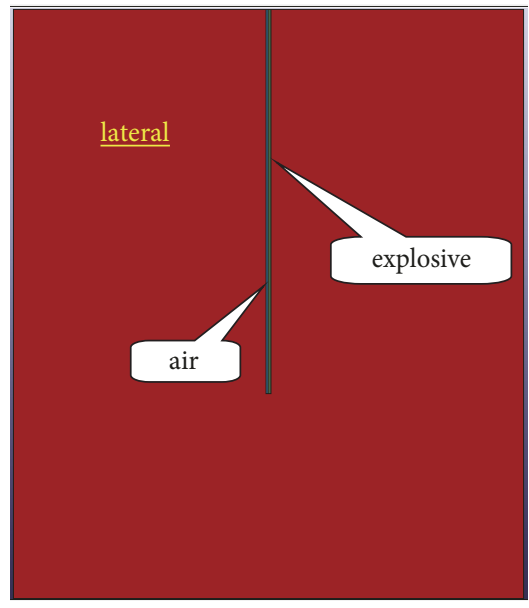

(a) Condition of air medium charge with eccentric decoupling coefficient of 2

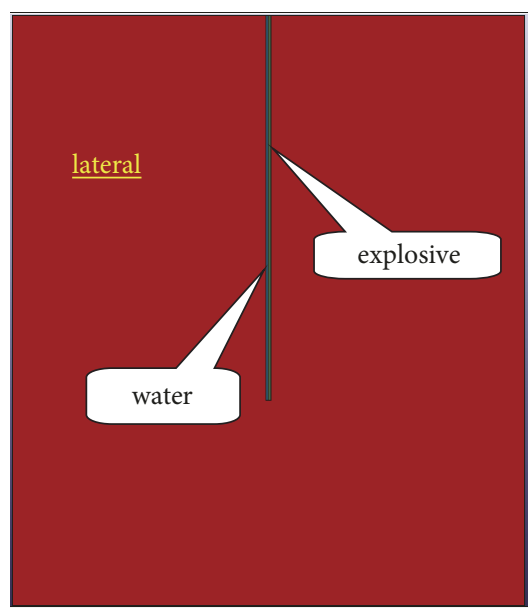

(c) Condition of water medium charge with eccentric decoupling coefficient of 2

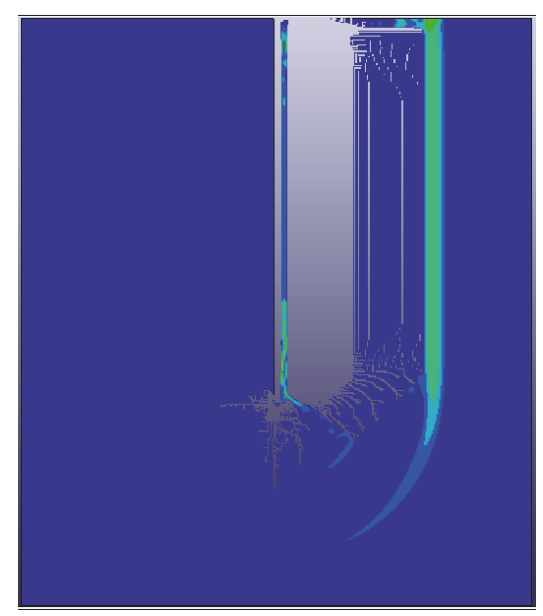

(b) Simulation results of charge by air medium

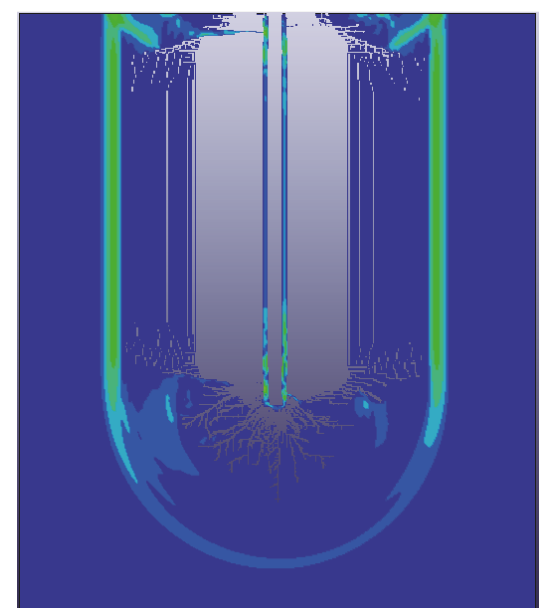

(d) Simulation results of charge by water medium

FIGURE 8: Numerical simulation results of eccentric decouple charge.

air. $E_{0}$ is internal energy and is taken as 0 for both water and air. $C, S_{1}, S_{2}$, and $S_{3}$ are all constants. $a$ is the first-order correction quantity of $\gamma_{0}$ and $\mu$, and $\mu=\rho / \rho_{0}-1$.

4.2. Calculation Model of Rock Materials. In the present study, the elastoplastic model $*$ MAT_PLASTIC_KINEMATIC [26], which is shown as (14), is adopted. The rock parameters of the model are introduced as follows: density $\rho_{c}$ is $3.8 \times$ $10^{3} \mathrm{~kg} / \mathrm{m}^{3}$, elastic modulus $E=25 \mathrm{GPa}$, and Poisson's ratio is 0.2 . The tangent modulus $E_{t}=1.5 \mathrm{G}$, hardening parameter $\varsigma=0.5$, and failure strain $\varepsilon=3.5$. The calculating constants $P$ and $C$ are taken as 4.0 and 2.5 , respectively.

$$
\sigma=\left[1+\left(\frac{\varepsilon}{C}\right)^{1 / P}\right]\left(\sigma_{0}+\varsigma \frac{E_{t} E}{E-E_{t}} \varepsilon_{e p}\right) .
$$

Figure 8 is a numerical simulation result of decoupled air medium and water medium charge condition when the eccentric coefficient is 2 . It is easy to find that the radius of the rock fracture zone for water medium decoupling charge is larger than that for air medium decoupling charge, suggesting that blast synergistic effect of water decoupling charge is more obvious than that for air decoupling charge.

Table 1 shows the specific data of the rock fracture radius for the numerical simulation results and corresponding theoretical solution. It can be seen that the theoretical and numerical results are in line with each other, and the deviation is less than $15 \%$. Therefore, it can be believed that the proposed eccentric decoupled numerical model is in good agreement with the theoretical study, which may have certain significance in studying the effect of eccentric decoupling charge.

\subsection{Study on the Influence of Eccentric Decouple Charge} Factor on Overexcavation of Surrounding Rock. Table 2 shows the numerical simulation results of the lateral fracture zone for the surrounding rock mass, and Figure 9 is the specific variation law of the fracture zone radius for the surrounding rock as the radial decoupling coefficient $k_{r}$ varies. Obviously, when the charging structure, the surrounding rock grade, 
TABLE 1: Comparison of theoretical solution and numerical solution for eccentric decouple charge.

\begin{tabular}{lcccc}
\hline \multirow{2}{*}{ decoupling medium } & wall rock & \multicolumn{2}{c}{ radius of broken rock zone $r_{c}(\mathrm{~cm})$} & deviation $( \pm \%)$ \\
& & theory solution & 2 & 0 \\
air & lateral & 2 & 60 & 15.0 \\
& medial & 52.16 & 68 & 12.6 \\
\multirow{2}{*}{ water } & lateral & 76.6 & 54 & 3.5 \\
\hline
\end{tabular}

TABLE 2: Numerical simulation results of the lateral fracture zone for the surrounding rock mass.

\begin{tabular}{|c|c|c|c|c|c|c|}
\hline \multirow{2}{*}{ Radial decoupling coefficient } & \multicolumn{5}{|c|}{ radius of broken lateral rock zone $(/ \mathrm{cm})$} & \multirow{2}{*}{ Surrounding rock grade } \\
\hline & RQD $=10 \mathrm{y} \%$ & $\mathrm{RQD}=35 \%$ & $\mathrm{RQD}=60 \%$ & RQD $=85 \%$ & $\mathrm{RQD}=95 \%$ & \\
\hline \multirow{3}{*}{4} & -- & -- & 2 & 2 & 2 & II \\
\hline & -- & 2 & 2 & 2 & -- & III \\
\hline & 2 & 2 & 2 & -- & -- & IV \\
\hline \multirow{3}{*}{2} & -- & -- & 2 & 2 & 2 & II \\
\hline & -- & 2 & 2 & 2 & -- & III \\
\hline & 2 & 2 & 2 & -- & -- & IV \\
\hline \multirow{3}{*}{1.33} & -- & -- & 33 & 24 & 22 & II \\
\hline & -- & 30 & 31 & 23 & -- & III \\
\hline & 35 & 32 & 32 & -- & -- & IV \\
\hline \multirow{3}{*}{1} & -- & -- & 60 & 52 & 50 & II \\
\hline & -- & 45 & 52 & 52 & -- & III \\
\hline & 40 & 49 & 39 & -- & -- & IV \\
\hline
\end{tabular}

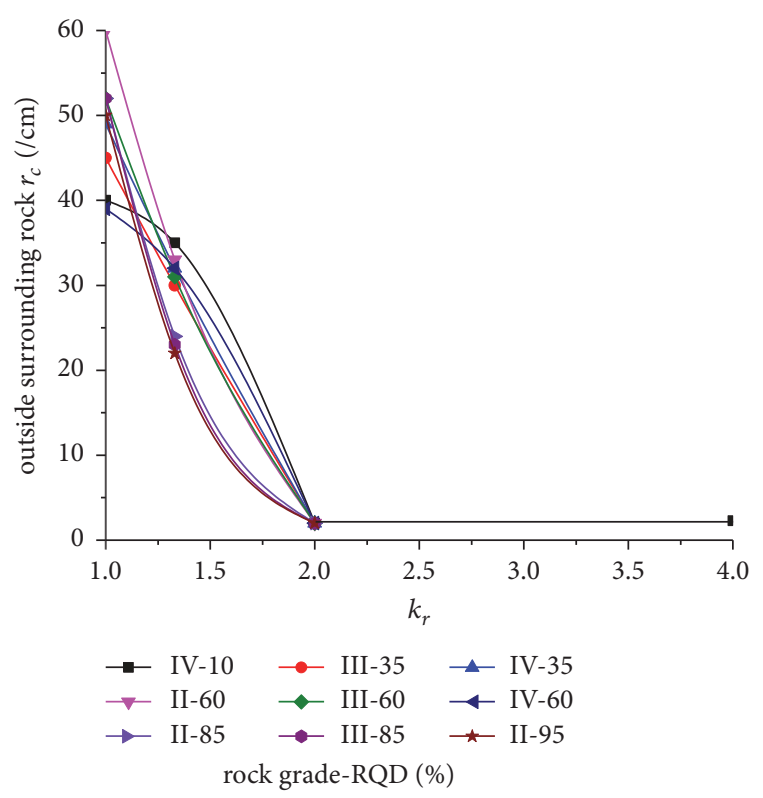

FIGURE 9: Relationship between $r_{c}$ of outside surrounding rock and $k_{r}$.

and the RQD value are the same, numerical analysis of each group shows that, with the increase of the radial coupling coefficient, the lateral radius of fractured zone takes on a decreasing tendency. Also, when the eccentric decoupling coefficient is larger than or equal to 2 , the radius of the lateral rock rupture zone is stabilized around the value of the blasthole radius. In other words, there is no overexcavation outside the surrounding rock.

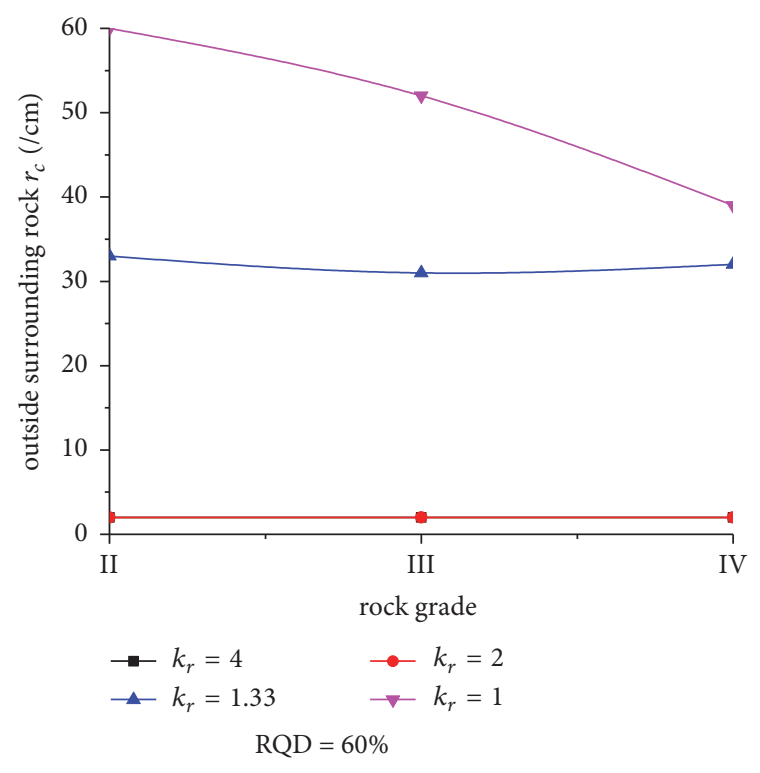

FIGURE 10: Relationship between fracture zone radius of outside surrounding rock $\left(r_{c}\right)$ and rock grade.

From the above, it can be concluded that under the precondition of no underexcavation, the charging structure with the eccentric decoupling coefficient larger than or equal to 2 should be possibly adopted.

4.4. Study about the Relation between the Fracture Zone Radius of Outside Surrounding Rock and the Rock Grade. From Table 2 and, in accordance with Figure 10, which is the 


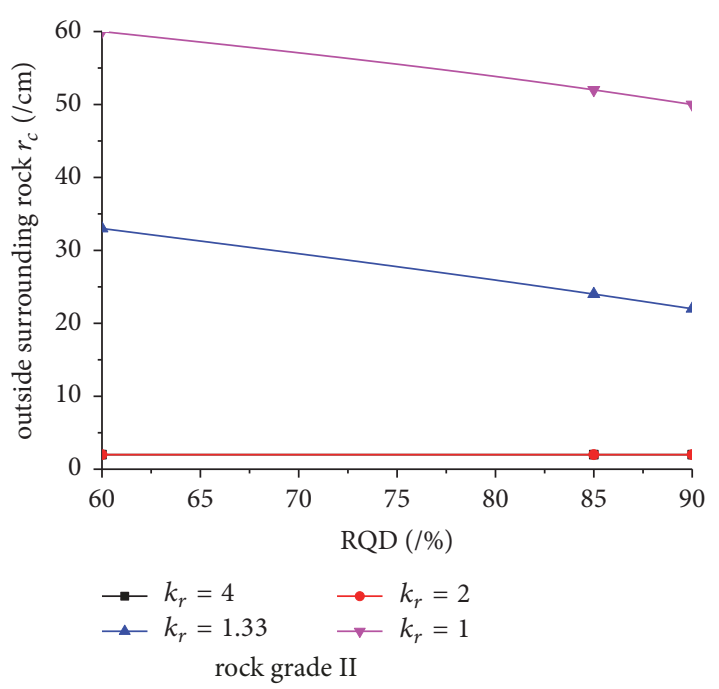

(a) Surrounding rock of grade II

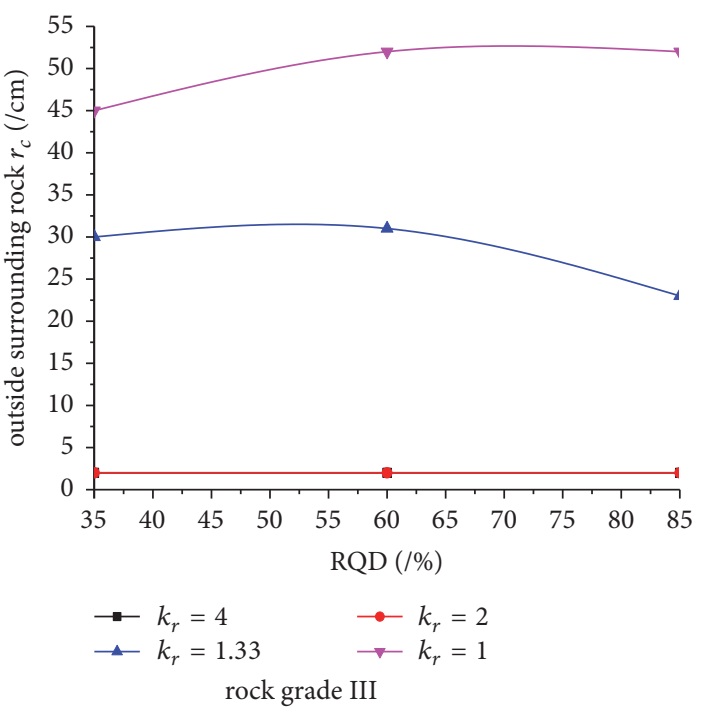

(b) Surrounding rock of grade III

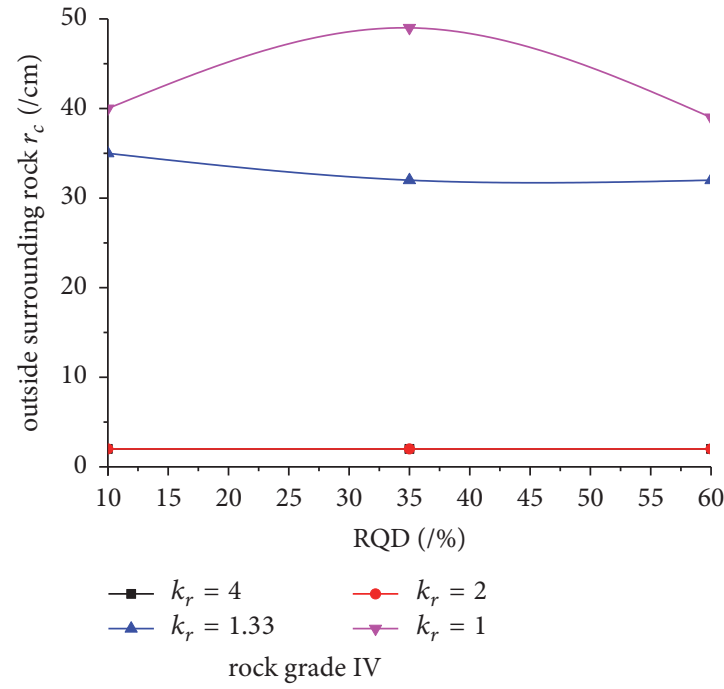

(c) Surrounding rock of grade IV

FIGURE 11: Relationship between fracture zone radius of outside surrounding rock $\left(r_{c}\right)$ and RQD value.

relationship between the fracture zone radius of outside surrounding rock $\left(r_{c}\right)$ and rock grade, it can be obtained from the numerical simulations that when the charge structure, radial eccentric coupling coefficient, and RQD value are in the same condition, the surrounding rock lateral radius of fractured zone decreases or keeps constant as the surrounding rock grade decreases.

4.5. Study on the Effect of Different RQD Values on the Blasting Effect. From the Table 2, the variation law between the fracture zone radius of outside surrounding rock $\left(r_{c}\right)$ and the RQD value of the surrounding rock can be established, which is depicted as Figure 11. It can be seen that, as the RQD value increases, $r_{c}$ shows a decreasing tendency for surrounding rock of grade II, whereas, for surrounding rock of grades III and IV, $r_{c}$ increases slightly at first and then decrease and finally keeps constant. The results suggest that the grade of the surrounding rock take an obvious effect on the relation between fracture zone radius of outside surrounding rock and RQD value.

\section{Conclusions}

In the present study, the analytical calculation model of eccentric decoupling charge is established based on the eccentric decoupled charge form on site and the concentric decoupled computational theory. Moreover, the influence factors including damage to the surrounding rock, air, and water decoupled media are taken into consideration in the present calculation model. Using ANSYS LS-DYNA, the proposed calculation model is applied to a case study. Finally, the following conclusions are obtained: 
(1) Comparing with the theoretical model, we can learn that the eccentric decoupling charge of air medium has better control of the overbreak-underbreak effect of the periphery hole than the eccentric uncoupling charge of water medium.

(2) Under the precondition of no underexcavation, the charging structure with the eccentric decoupling coefficient larger than or equal to 2 should be possibly adopted.

(3) Under the same condition of other charging parameters, the radius of the fracture area of surrounding rock decreases with the decrease of the surrounding rock grade, and the change is always decreasing or stable.

(4) Under the same condition of other charging parameters, the radius of the fracture area of surrounding rock increases with the increase of RQD value of surrounding rock. The determination of specific charge parameters can be determined by the numerical simulation diagram according to the actual conditions.

\section{Conflicts of Interest}

The authors declare that there are no conflicts of interest regarding the publication of this paper.

\section{References}

[1] W. L. Fourney, J. W. Dally, and D. C. Holloway, "Controlled blasting with ligamented charge holders," International Journal of Rock Mechanics and Mining Sciences, vol. 15, no. 3, pp. 184$188,1978$.

[2] V. K. Singh and D. P. Singh, "Controlled blasting in an open-pit mine for improved slope stability," Geotechnical and Geological Engineering, vol. 13, no. 1, pp. 51-57, 1995.

[3] E. Deng, W. Yang, M. Lei, R. Yin, and P. Zhang, "Instability Mode Analysis of Surrounding Rocks in Tunnel Blasting Construction with Thin Bedrock Roofs," Geotechnical and Geological Engineering, pp. 1-12, 2018.

[4] C. H. Dowding and C. T. Aimone, "Multiple blast-hole stresses and measured fragmentation," Rock Mechanics and Rock Engineering, vol. 18, no. 1, pp. 17-36, 1985.

[5] U. Langefors and B. Kihstrom, The Modern Technique of Rock Blasting, Wiley, NY, USA, 1963.

[6] G. W. Ma and X. M. An, "Numerical simulation of blastinginduced rock fracture," International Journal of Rock Mechanics and Mining Sciences, vol. 45, pp. 966-975, 2008.

[7] S. H. Cho, Y. Nakamura, B. Mohanty, H. S. Yang, and K. Kaneko, "Numerical study of fracture plane control in laboratory-scale blasting," Engineering Fracture Mechanics, vol. 75, no. 13, pp. 3966-3984, 2008.

[8] S. K. Mandal, M. M. Singh, and S. Dasgupta, "Theoretical concept to understand plan and design smooth blasting pattern," Geotechnical and Geological Engineering, vol. 26, no. 4, pp. 399416, 2008.

[9] Y. B. Wang, "Study of the dynamic fracture effect using slotted cartridge decoupling charge blasting," International Journal of Rock Mechanics \& Mining Sciences, vol. 18, pp. 34-36, 2017.

[10] Y. Z. Wei, "A new theory about the effect of unsymmetrical explosive loading in a borehole on rock fracture," Journal of Jiangxi Institute Of Metallurgy, vol. 7, no. 2, pp. 63-75, 1986.

[11] D. S. Liu and S. L. Li, Blasting Works, Beijing Science Press, 2011.
[12] Z. Yu, D. N. Lin, and S. Wu, "On blasting characteristics and optimal explosive column position of water medium eccentric decouple charge blasting," Mineral Engineering Research, vol. 30, no. 3, pp. 1-4, 2015.

[13] C. J. Pu, "Test and research on eccentric decouple charge blasting," Industrial Minerals Processing, vol. 36, no. 4, pp. 3032, 2007.

[14] J. H. Zhang, "Stress- field research and application of eccentric decouple charge," Industrial Safety and Environment al Protection, vol. 27, no. 8, pp. 20-23, 2001.

[15] Z. C. Zhang, J. J. Shi, C. J. Pu et al., "Expermiental Research of Rock Damage by Eccentric Decouple Charge Blasting," Blasting, vol. 23, no. 4, pp. 4-8, 2006.

[16] S. H. Guan, C. J. Pu, and D. J. Xiao, "Experimental Study on Crack Propagation under Eccentric Decouple Charge Structure," Blasting, vol. 32, no. 1, pp. 16-21, 2015.

[17] J. J. Shi, Z. C. Zhang, Z. X. Xiao et al., “Testing study of rock damage under eccentric decouple charge blasting," Opencast Mining Technology, vol. 1, pp. 32-35, 2006.

[18] А. Н. Ханукаев, The Physical Process of Rock Blasting, Metallurgical Industry Press, Beijing, China, 1980.

[19] W. L. Fourney, D. B. Barker, and D. C. Holloway, "Model studies of explosive well stimulation techniques," International Journal of Rock Mechanics \& Mining Sciences \& Geomechanics Abstracts, vol. 18, no. 2, pp. 113-127, 1981.

[20] Q. Zong and D. Meng, "Influence of different kinds of hole charging structure on explosive energy transmission," Chinese Journal of Rock Mechanics and Engineering, vol. 22, no. 4, pp. 641-645, 2003.

[21] Q. Zong, L. Tian, H. B. Wang et al., "Study and Application on Rock Damage Range by Blasting with Water-decoupled Charge," Blasting, vol. 29, no. 2, pp. 42-46, 2012.

[22] J.-H. Chen, J.-S. Zhang, and X.-P. Li, "Study of presplitting blasting parameters and its application based on rock blastinginduced damage theory," Rock and Soil Mechanics, vol. 37, no. 5, pp. 1441-1450, 2016.

[23] Y. Zhang, W. Lu, M. Chen, P. Yan, and J. Yang, "Rock cracking mechanism driven by explosive stress wave," Chinese Journal of Rock Mechanics and Engineering, vol. 33, no. 1, pp. 3144-3149, 2014.

[24] M. S. Zhong, Y. Long, X. H. Li et al., "Time function for borehole explosive loading and specific energy based on different coupling mediums," Journal of Vibration and Shock, vol. 30, no. 7, pp. 116-119, 2011.

[25] LSTC, LS-DYNA Keyword User's Manual, California: Livermore Software Technology Corporation, 2003.

[26] D. X. Ding and C. Z. Zhu, "Estimating the Amount of Explosives for Notched Borehole Blasting," Transactions of Nonferrous Metals Society of China, vol. 3, no. 2, pp. 18-21, 1993. 


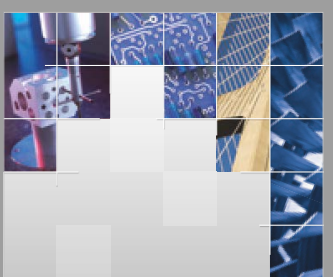

\section{Enfincering}
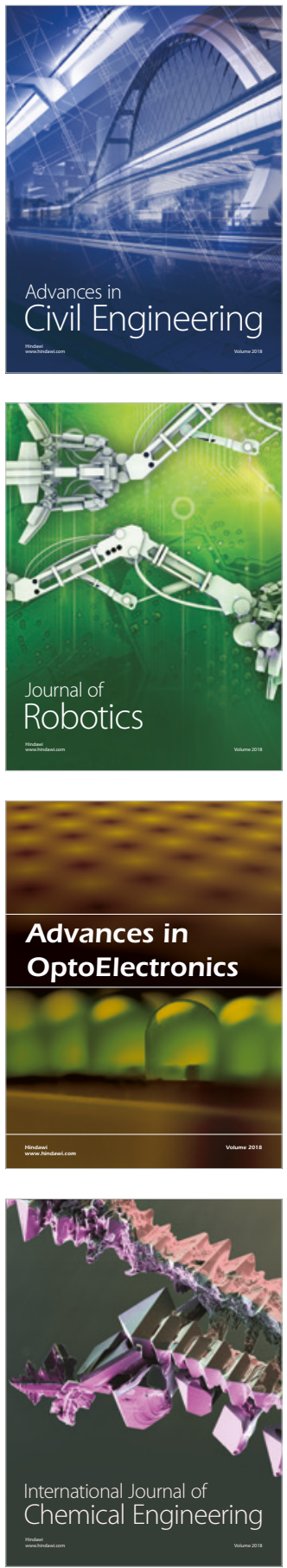

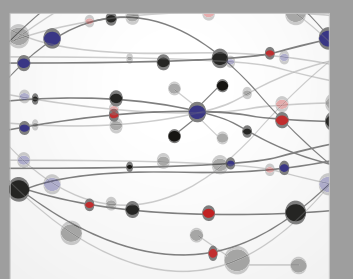

\section{Rotating \\ Machinery}

The Scientific World Journal

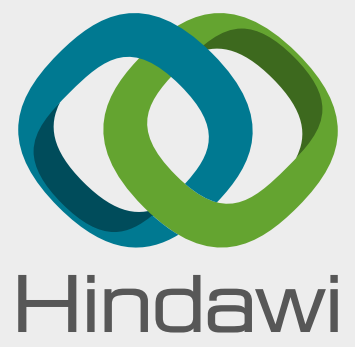

Submit your manuscripts at

www.hindawi.com
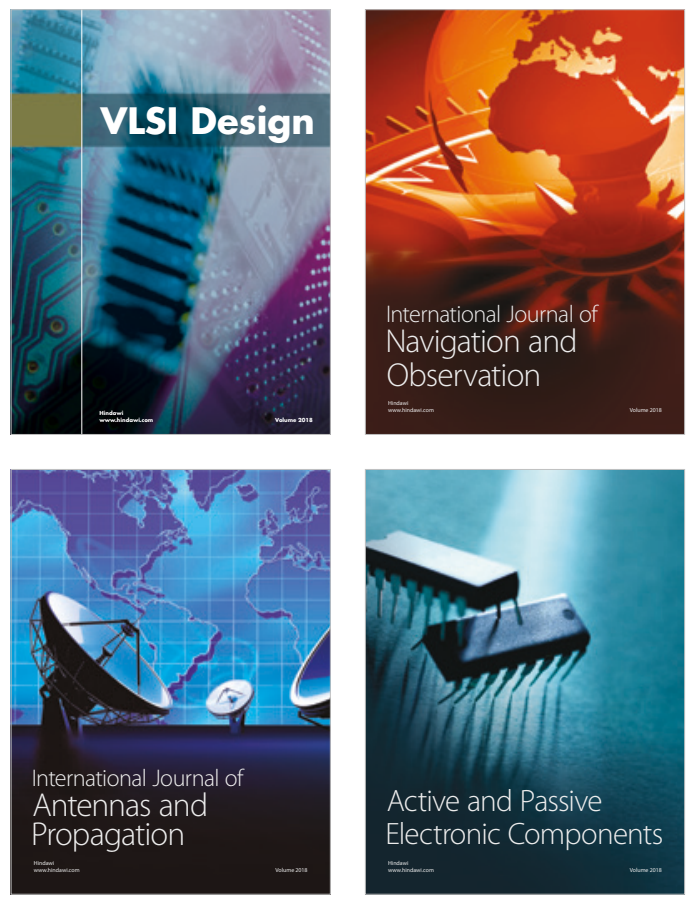
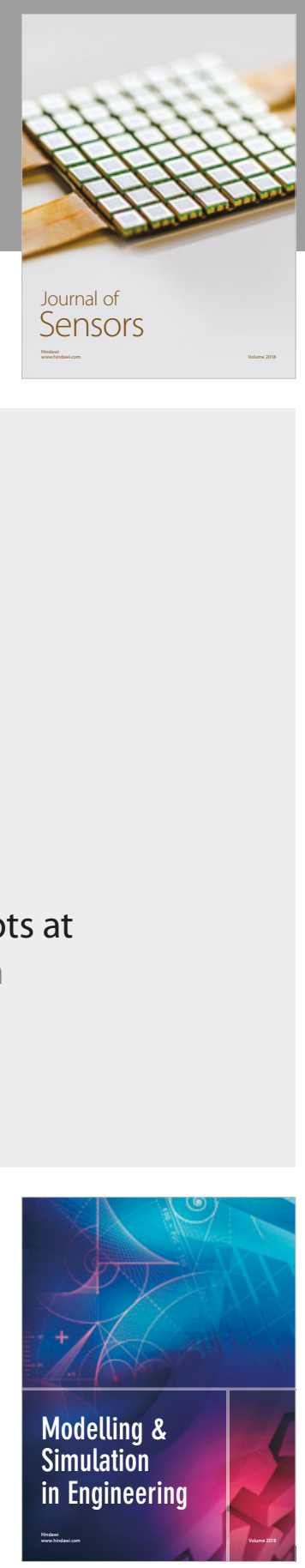

\section{Advances \\ Multimedia}
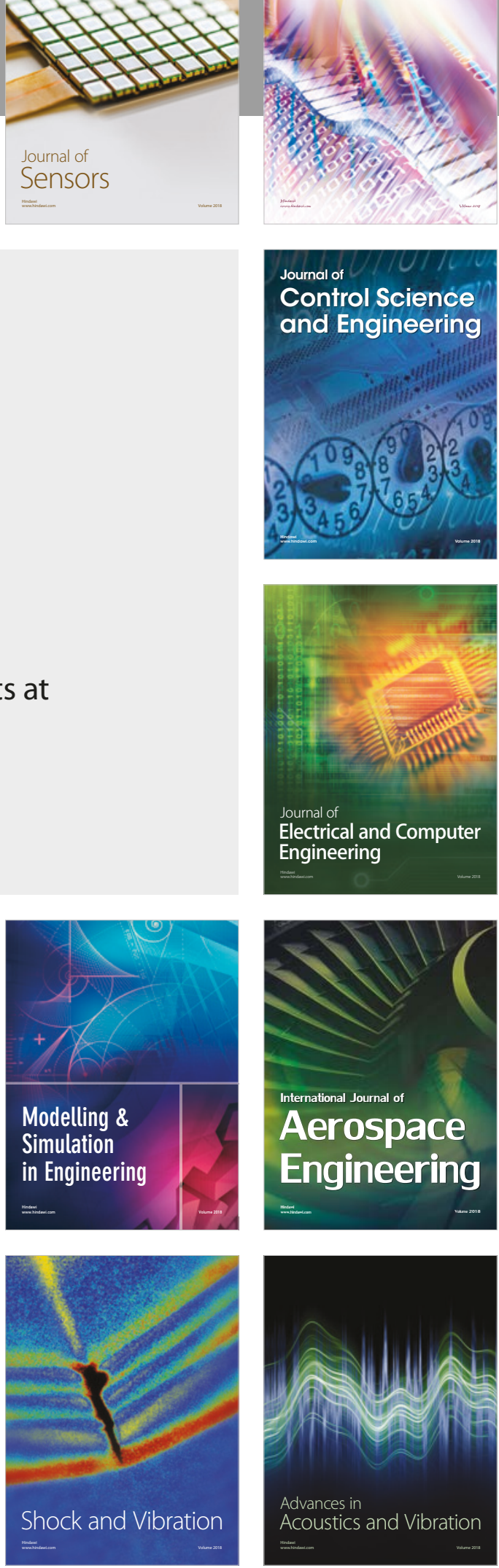